\title{
Survival in Patients with Pyrrolizidine Alkaloids-Induced Hepatic Sinusoidal Obstruction Syndrome Versus Other Causes of Portal Hypertension in the Treatment of Transjugular Intrahepatic Portosystemic Shunt Placement: A Propensity Score Matching Study
}



\section{Research Article}

Keywords: Autoimmune hepatitis, Cryptogenic chronic hepatitis, Hepatic sinusoidal obstruction syndrome, Hepatic transplantation, Primary biliary cirrhosis, Pyrrolizidine alkaloids, Schistosomiasis, Outcomes assessment, Survival analysis, Viral hepatitis, Human

Posted Date: February 2nd, 2021

DOI: https://doi.org/10.21203/rs.3.rs-152407/v1

License: (c) (i) This work is licensed under a Creative Commons Attribution 4.0 International License. Read Full License 


\section{Abstract}

Background/purpose: The etiology of portal hypertension may be an important factor affecting patient outcomes after transjugular intrahepatic portosystemic shunt (TIPS) treatment. Pyrrolizidine alkaloid-induced hepatic sinusoidal obstruction syndrome (PA-HSOS) is characterized by acute portal hypertension and liver injury. The aim of this study was to compare the post-TIPS survival between patients with PA-HSOS and those with other etiologies of hepatic portal hypertension.

Methods: A retrospective file review of 787 patients with portal hypertension-related complications, including 146 patients with PA-HSOS, who had received TIPS treatment at our institution between August 2008 to September 2020 was performed. We recorded the demographic and clinical characteristics of patients before TIPS. The median follow-up was 20.27 months. Orthotopic liver transplantation (OLT)-free survival was compared between PA-HSOS and non-HSOS patients. Propensity score matching (PSM) was used to minimize bias.

Results: Compared to patients with other etiologies of portal hypertension, PA-HSOS patients had a statistically significantly higher Child-Pugh and model for end-stage liver disease score. No significant differences were found in the orthotopic liver transplantation (OLT)-free survival between patients with PA-HSOS and patients with other causes of portal hypertension (all $P>0.05$ ). After PSM, OLT-free survival was significantly better in PA-HSOS patients than in patients with viral hepatitis $(P=0.0011)$, primary biliary cirrhosis or autoimmune hepatitis $(P=0.021)$, and schistosomiasis $(P=0.025)$.

Conclusions: TIPS placement can be performed safely in patients with PA-HSOS. PA-HSOS patients had a better or similar prognosis after TIPS treatment than patients with portal hypertension of other etiologies.

Trial registration: This study was retrospectively registered in the chictr.Org database (ChiCTR2000040899) in 14 December 2020.

\section{Introduction}

The hepatic sinusoidal obstruction syndrome (HSOS), also known as hepatic veno-occlusive disease (HVOD), is a hepatic vascular disease characterized by swelling and necrosis of sinusoidal endothelial cells in zone 3 of the hepatic acini leading to sinusoidal congestion [1]. It is often associated with hematopoietic stem cell transplantation, the use of certain chemotherapeutic agents in non-transplant conditions (drug-induced liver injury), and immunosuppressive agents after liver transplantation in Western countries [2-4]. In China, the leading cause of HSOS is the intake of herbs containing pyrrolizidine alkaloids (PA) [5-6]. There are at least 6,000 plants worldwide that contain PAs, with the most common being Gynura segetum, in the genus Senecio of the family Compositae. Ingestion of Tusanqi contributes to about $50-86.6 \%$ of reported PA-HSOS in China [1].

Acute sinusoidal portal hypertension can occur in PA-HSOS because of the progressive narrowing and blocking of the sinusoidal and intrahepatic venous lumen and may further aggravate liver damage [7]. A transjugular intrahepatic portosystemic shunt (TIPS) is an important treatment of portal hypertension-related complications [8]. To date, anticoagulant and supportive treatment is the first-line treatment for HSOS based on current consensus [1], but the response rate is only approximately $60 \%$ [9-11]. In patients who do not respond to medication, TIPS is recommended as a second-line salvage therapy, even though this remains controversial [1].

The literature currently provides no data comparing the long-term survival of PA-HSOS patients with that of patients with other etiologies of portal hypertension after TIPS treatment. Given the unique etiology and mechanism of sinusoidal portal hypertension in PA-HSOS patients and its high prevalence in China, We hypothsized that the different etiologies of portal hypertension with their underlying pathogenic mechanisms may result in different long-term survival after TIPS.

Therefore, the aim of this study was to compare the survival of patients with PA-HSOS with that of patients with portal hypertension caused by other diseases after TIPS treatment.

\section{Material And Methods \\ Patients and data collection}

A retrospective review of the medical records of 1011 patients diagnosed with portal hypertension and successfully underwent TIPS at our center from August 2008 to September 2020 was performed. The exclusion criteria were as follows: 1) incomplete data; 2) severe, life-threatening disease of another system unrelated to portal hypertension and its complications; 3) previous TIPS or surgical shunting; 4) hepatocellular carcinoma before TIPS; and 5) follow-up time after TIPS therapy of fewer than six months. A total of 224 patients were excluded based on these criteria. Finally, 787 patients were enrolled. The patient flow chart is shown in Fig. 1.

The diagnosis of PA-HSOS was made according to the Nanjing criteria [1], which include a history of ingestion of a PA-containing plant and meeting of the following three criteria or pathological evidence that rules out other known causes of liver injury: abdominal distention and/or pain 
in the hepatic region, hepatomegaly, and ascites; elevation of total serum bilirubin or abnormal liver function testing; and evidence on enhanced computed tomography or magnetic resonance imaging, such as a map-like or mottle-like nonhomogeneous appearance in the venous phase and equilibrium phase and a halo sign around the portal vein. Typical pathological changes of HSOS include swelling, damage, and shedding of sinusoidal endothelial cells in the acinus zone 3 and significant dilation and congestion of the sinusoids.

The diagnostic criteria for other etiologies of portal hypertension were based on the following criteria [12-18 ]. A mixed etiology of portal hypertension was defined as the combination of no less than two etiologies. The diagnostic criteria for portal hypertension were based on the Baveno VI consensus in all patients [19]. All methods were carried out in accordance with relevant guidelines and regulations.

\section{TIPS procedure}

TIPS procedures were conducted by an experienced team using the standard techniques described previously [20-21]. Briefly, the Rups-100 device (Rösch-Uchida Transjugular Liver Access Set with Check-Flo® Introducer, Cook Medical Inc., Bloomington, IN, USA) was introduced via the right jugular vein into the inferior vena cava and hepatic vein. After successfully passing the portal vein, a composite of a covered stent (Fluency ${ }^{\mathrm{TM}}$; Bard Peripheral Vascular Inc., Tempe, AZ, USA) combined with a bare stent (E-Luminexx ${ }^{\mathrm{TM}}$; Bard Peripheral Vascular Inc., Tempe, AZ, USA) or a Viatorr ${ }^{\circledR}$ stent (W. L. Gore \& Associates, Inc., Flagstaff, AZ, USA) was deployed. The stent diameters used were $6 \mathrm{~mm}, 7 \mathrm{~mm}$, and $8 \mathrm{~mm}$. In addition, any varicose collateral veins visible on angiography were embolized using tissue glue and/or coils during the TIPS procedure.

\section{Statistical analyses}

Quantitative variables with normal distribution were expressed as the mean and standard deviation and compared using the Student's t-test. Variables that were not normally distributed were presented as the median and range, and the Mann-Whitney-Wilcoxon test was used for comparisons. Categorical variables were described as numbers and frequencies and compared using Pearson's Chi-square or Fisher's exact tests.

Survival rates were analyzed using the Kaplan-Meier model. Univariate analysis was used to assess a potential influence of patient characteristics on the prognosis. Statistically significant factors were introduced into a multivariate Cox proportional hazards analysis. All tests were two-sided, and a $P$-value $<0.05$ was defined as statistically significant.

All missing data were imputed using the E-M model. Sex, age, the model for end-stage liver disease (MELD) score, CTP score, stent diameter, and pre-TIPS episodes of overt hepatic encephalopathy (OHE) were used as matching factors to adjust for baseline differences, and the selection was performed without replacement. Propensity score matching may become more difficult with more variables, but these variables may be the most critical ones with regard to outcomes.

The R software, version 3.5.1 (http://www.r-project.org) was used for all analyses within a range of 0.2 of the standard deviation.

\section{Results}

\section{Baseline characteristics of the study population}

The baseline characteristics of all patients PA-HSOS and non-PA HSOS are summarized in Table 1. Nearly half of the study population consisted of hepatitis-B virus (HBV) patients $(n=366)$, followed by PA-HSOS patients $(n=146)$. Patients with a mixed etiology of liver cirrhosis were rare in our study $(n=14)$. In general, PA-HSOS patients had more severe liver injury as reflected by a significantly higher CTP score and MELD score than all other patients except for HBV patients. The proportion of patients with ascites among PA-HSOS patients was higher than that among patients with all other etiologies except for HBV. PA-HSOS patients had a markedly lower portal vein velocity (PVV) than patients with all other etiologies except for a mixed etiology. The reduction of the portal pressure gradient compared to the intraoperative values was significantly higher in PA-HSOS patients than in patients with all other causes of portal hypertension except for cryptogenic portal hypertension. 
Table 1

Baseline demographic and clinical characteristics of 787 patients before transjugular intrahepatic portosystemic shunt placement.

$\begin{array}{lllllll}\text { PA-HSOS HBV } & \text { Alcohol PBC } & \text { AlH } & \text { Schistosomiasis HCV } & \text { Mixed }\end{array}$

$(n=146) \quad(n=366) \quad(n=69) \quad(n=64) \quad(n=30) \quad(n=27) \quad(n=29) \quad(n=14) \quad(n=42)$

Demographic and clinical

characteristics

\begin{tabular}{|c|c|c|c|c|c|c|c|c|c|}
\hline Male gender & $\begin{array}{l}95 \\
(65.07 \%)\end{array}$ & $\begin{array}{l}284 \\
(77.60 \%)\end{array}$ & $\begin{array}{l}68 \\
(98.55 \%)\end{array}$ & $\begin{array}{l}9 \\
(14.06 \%)\end{array}$ & $\begin{array}{l}0 \\
(0.00 \%)\end{array}$ & 16 (59.26\%) & $14(48.28 \%)$ & $\begin{array}{l}12 \\
(85.71 \%)\end{array}$ & $\begin{array}{l}16 \\
(38.10 \%)\end{array}$ \\
\hline$P$ value & & 0.004 & 0 & 0 & 0 & 0.563 & 0.088 & 0.145 & 0.002 \\
\hline Age (years) & $\begin{array}{l}63.69 \pm \\
9.63\end{array}$ & $\begin{array}{l}53.00 \pm \\
11.26\end{array}$ & $\begin{array}{l}58.57 \pm \\
10.15\end{array}$ & $\begin{array}{l}58.84 \pm \\
9.98\end{array}$ & $\begin{array}{l}62.80 \pm \\
8.30\end{array}$ & $63.41 \pm 13.71$ & $60.21 \pm 9.75$ & $\begin{array}{l}51.00 \pm \\
12.54\end{array}$ & $\begin{array}{l}63.55 \pm \\
10.88\end{array}$ \\
\hline$P$ value & & 0.001 & 0 & 0.001 & 0.637 & 0.896 & 0.077 & 0 & 0.934 \\
\hline MELD score & $\begin{array}{l}14.34 \pm \\
6.48\end{array}$ & $\begin{array}{l}9.92 \pm \\
2.23\end{array}$ & $\begin{array}{l}10.74 \pm \\
3.19\end{array}$ & $\begin{array}{l}9.06 \pm \\
2.09\end{array}$ & $\begin{array}{l}9.21 \pm \\
2.28\end{array}$ & $9.88 \pm 2.85$ & $9.93 \pm 2.30$ & $\begin{array}{l}9.21 \pm \\
2.94\end{array}$ & $9.74 \pm 2.72$ \\
\hline$P$ value & & 0 & 0 & 0 & 0 & 0 & 0 & 0 & 0 \\
\hline
\end{tabular}

Child-Pugh score

\begin{tabular}{|c|c|c|c|c|c|c|c|c|c|}
\hline$A(5-6)$ & $\begin{array}{l}3 \\
(2.05 \%)\end{array}$ & $\begin{array}{l}123 \\
(33.61 \%)\end{array}$ & $\begin{array}{l}13 \\
(18.84 \%)\end{array}$ & $\begin{array}{l}16 \\
(25.00 \%)\end{array}$ & $\begin{array}{l}9 \\
(30.00 \%)\end{array}$ & $6(22.22 \%)$ & $11(37.93 \%)$ & $\begin{array}{l}4 \\
(28.57 \%)\end{array}$ & $\begin{array}{l}15 \\
(35.71 \%)\end{array}$ \\
\hline $\mathrm{B}(7-9)$ & $\begin{array}{l}76 \\
(52.05 \%)\end{array}$ & $\begin{array}{l}226 \\
(61.75 \%)\end{array}$ & $\begin{array}{l}54 \\
(78.26 \%)\end{array}$ & $\begin{array}{l}47 \\
(73.44 \%)\end{array}$ & $\begin{array}{l}21 \\
(70.00 \%)\end{array}$ & $20(74.07 \%)$ & 17 (58.62\%) & $\begin{array}{l}9 \\
(64.29 \%)\end{array}$ & $\begin{array}{l}26 \\
(61.90 \%)\end{array}$ \\
\hline$C(10-13)$ & $\begin{array}{l}67 \\
(45.89 \%)\end{array}$ & $\begin{array}{l}17 \\
(4.64 \%)\end{array}$ & $\begin{array}{l}2 \\
(2.90 \%)\end{array}$ & $\begin{array}{l}1 \\
(1.56 \%)\end{array}$ & $\begin{array}{l}0 \\
(0.00 \%)\end{array}$ & $1(3.70 \%)$ & $1(3.45 \%)$ & 1 (7.14\%) & $1(2.38 \%)$ \\
\hline$P$ value & & 0.509 & 0 & 0 & 0 & 0 & 0 & 0 & 0 \\
\hline
\end{tabular}

Bleeding episodes

\begin{tabular}{|c|c|c|c|c|c|c|c|c|c|}
\hline Absent & $\begin{array}{l}144 \\
(98.63 \%)\end{array}$ & $\begin{array}{l}21 \\
(5.74 \%)\end{array}$ & $\begin{array}{l}9 \\
(13.04 \%)\end{array}$ & $\begin{array}{l}2 \\
(3.13 \%)\end{array}$ & $\begin{array}{l}2 \\
(6.67 \%)\end{array}$ & 4 (14.81\%) & $2(6.90 \%)$ & $\begin{array}{l}2 \\
(14.29 \%)\end{array}$ & $1(2.38 \%)$ \\
\hline Once & $\begin{array}{l}2 \\
(1.37 \%)\end{array}$ & $\begin{array}{l}85 \\
(23.22 \%)\end{array}$ & $\begin{array}{l}22 \\
(31.88 \%)\end{array}$ & $\begin{array}{l}18 \\
(28.13 \%)\end{array}$ & $\begin{array}{l}11 \\
(36.67 \%)\end{array}$ & $3(11.11 \%)$ & $7(24.14 \%)$ & $\begin{array}{l}3 \\
(21.43 \%)\end{array}$ & $6(14.29 \%)$ \\
\hline Multiple & $\begin{array}{l}0 \\
(0.00 \%)\end{array}$ & $\begin{array}{l}260 \\
(71.04 \%)\end{array}$ & $\begin{array}{l}38 \\
(55.07 \%)\end{array}$ & $\begin{array}{l}44 \\
(68.75 \%)\end{array}$ & $\begin{array}{l}17 \\
(56.67 \%)\end{array}$ & $20(74.07 \%)$ & $20(68.97 \%)$ & $\begin{array}{l}9 \\
(64.29 \%)\end{array}$ & $\begin{array}{l}35 \\
(83.33 \%)\end{array}$ \\
\hline$P$ value & & 0.002 & 0 & 0 & 0 & 0 & 0 & 0 & 0 \\
\hline \multicolumn{10}{|l|}{ Ascites } \\
\hline Absent & $\begin{array}{l}0 \\
(0.00 \%)\end{array}$ & $\begin{array}{l}103 \\
(28.14 \%)\end{array}$ & $\begin{array}{l}10 \\
(14.49 \%)\end{array}$ & $\begin{array}{l}11 \\
(17.19 \%)\end{array}$ & $\begin{array}{l}7 \\
(23.33 \%)\end{array}$ & 5 (18.52\%) & 9 (31.03\%) & $\begin{array}{l}4 \\
(28.57 \%)\end{array}$ & $\begin{array}{l}11 \\
(26.19 \%)\end{array}$ \\
\hline Mild & $\begin{array}{l}1 \\
(0.68 \%)\end{array}$ & $\begin{array}{l}118 \\
(32.24 \%)\end{array}$ & $\begin{array}{l}26 \\
(37.68 \%)\end{array}$ & $\begin{array}{l}20 \\
(31.25 \%)\end{array}$ & $\begin{array}{l}8 \\
(26.67 \%)\end{array}$ & 8 (29.63\%) & 9 (31.03\%) & $\begin{array}{l}5 \\
(35.71 \%)\end{array}$ & $9(21.43 \%)$ \\
\hline Moderate & $\begin{array}{l}49 \\
(33.56 \%)\end{array}$ & $\begin{array}{l}108 \\
(29.51 \%)\end{array}$ & $\begin{array}{l}20 \\
(28.99 \%)\end{array}$ & $\begin{array}{l}24 \\
(37.50 \%)\end{array}$ & $\begin{array}{l}13 \\
(43.33 \%)\end{array}$ & 9 (33.33\%) & 11 (37.93\%) & $\begin{array}{l}4 \\
(28.57 \%)\end{array}$ & $\begin{array}{l}17 \\
(40.48 \%)\end{array}$ \\
\hline Massive & $\begin{array}{l}96 \\
(65.75 \%)\end{array}$ & $\begin{array}{l}37 \\
(10.11 \%)\end{array}$ & $\begin{array}{l}13 \\
(18.84 \%)\end{array}$ & $\begin{array}{l}9 \\
(14.06 \%)\end{array}$ & $\begin{array}{l}2 \\
(6.67 \%)\end{array}$ & $5(18.52 \%)$ & $0(0.00 \%)$ & $1(7.14 \%)$ & $5(11.90 \%)$ \\
\hline$P$ value & & 0.091 & 0 & 0 & 0 & 0 & 0 & 0 & 0 \\
\hline \multicolumn{10}{|c|}{ Laboratory tests } \\
\hline \multirow[t]{2}{*}{ PT (s) } & 16.10 & 14.70 & 14.40 & 13.60 & 13.45 & 13.50 & 14.50 & 14.00 & 14.10 \\
\hline & $\begin{array}{l}(14.00- \\
18.40)\end{array}$ & $\begin{array}{l}(13.50- \\
16.00)\end{array}$ & $\begin{array}{l}(13.20- \\
16.60)\end{array}$ & $\begin{array}{l}(12.45- \\
14.70)\end{array}$ & $\begin{array}{l}(12.70- \\
14.60)\end{array}$ & $(12.40-14.40)$ & $(13.60-15.70)$ & $\begin{array}{l}(12.50- \\
14.60)\end{array}$ & $\begin{array}{l}(12.90- \\
15.00)\end{array}$ \\
\hline$P$ value & & 0 & 0 & 0 & 0 & 0 & 0.004 & 0.001 & 0 \\
\hline
\end{tabular}

Abbreviations: AlH, autoimmune hepatitis; ALT, alanine aminotransferase; AST, aspartate aminotransferase; Cryptogenic, cryptogenic liver disease; HBV, hepatitis B virus; HCV, hepatitis C virus; INR, international normalized ratio; MELD, model for end-stage liver disease; OHE, overt hepatic encephalopathy; PA-HSOS, pyrrolizidine alkaloid-induced hepatic sinusoidal obstruction syndrome; PBC, primary biliary cirrhosis; PPG, portal pressure gradient; PT, prothrombin time; PVP, portal vein pressure; PVT, portal vein thrombosis; PVV, portal vein velocity; TBil, total bilirubin; TIPS, transjugular intrahepatic portosystemic shunt placement; WBC, white blood count. 


\begin{tabular}{|c|c|c|c|c|c|c|c|c|c|}
\hline & PA-HSOS & HBV & Alcohol & PBC & AlH & Schistosomiasis & $\mathrm{HCV}$ & Mixed & Cryptogenic \\
\hline \multirow[t]{2}{*}{ INR } & 1.42 & 1.28 & 1.26 & 1.19 & 1.16 & 1.17 & 1.25 & 1.23 & 1.24 \\
\hline & $\begin{array}{l}(1.22- \\
1.61)\end{array}$ & $\begin{array}{l}(1.18- \\
1.40)\end{array}$ & $\begin{array}{l}(1.14- \\
1.41)\end{array}$ & $\begin{array}{l}(1.09- \\
1.28)\end{array}$ & $\begin{array}{l}(1.11- \\
1.29)\end{array}$ & $(1.07-1.27)$ & $(1.18-1.35)$ & $\begin{array}{l}(1.09- \\
1.31)\end{array}$ & $(1.13-1.33)$ \\
\hline \multicolumn{2}{|l|}{$P$ value } & 0 & 0 & 0 & 0 & 0 & 0.002 & 0.004 & 0 \\
\hline \multirow[t]{2}{*}{$\operatorname{ALT}(\mathrm{U} / \mathrm{L})$} & 43.40 & \multirow{2}{*}{$\begin{array}{l}21.40 \\
(15.25- \\
28.15)\end{array}$} & 21.20 & \multirow{2}{*}{$\begin{array}{l}25.55 \\
(16.50- \\
36.70)\end{array}$} & \multirow{2}{*}{$\begin{array}{l}20.55 \\
(12.90- \\
40.50)\end{array}$} & \multirow{2}{*}{$\begin{array}{l}18.20 \\
(14.50-25.10)\end{array}$} & \multirow{2}{*}{$\begin{array}{l}21.70(16.00- \\
33.90)\end{array}$} & \multirow{2}{*}{$\begin{array}{l}20.60 \\
(14.30- \\
58.50)\end{array}$} & \multirow{2}{*}{$\begin{array}{l}19.90 \\
(14.40- \\
24.80)\end{array}$} \\
\hline & $\begin{array}{l}(22.20- \\
92.90)\end{array}$ & & $\begin{array}{l}(15.10- \\
33.70)\end{array}$ & & & & & & \\
\hline$P$ value & & 0 & 0 & 0 & 0.001 & 0 & 0 & 0.01 & 0 \\
\hline \multirow[t]{2}{*}{ AST $(\mathrm{U} / \mathrm{L})$} & 60.55 & 28.00 & 31.70 & 36.70 & 29.20 & 26.85 & 27.70 & 27.10 & 26.50 \\
\hline & $\begin{array}{l}(36.20- \\
115.30)\end{array}$ & $\begin{array}{l}(21.75- \\
37.45)\end{array}$ & $\begin{array}{l}(21.60- \\
46.70)\end{array}$ & $\begin{array}{l}(25.20- \\
56.80)\end{array}$ & $\begin{array}{l}(21.20- \\
56.80)\end{array}$ & $(22.20-32.50)$ & $(20.70-36.20)$ & $\begin{array}{l}(19.90- \\
44.80)\end{array}$ & $\begin{array}{l}(21.30- \\
36.80)\end{array}$ \\
\hline$P$ value & & 0 & 0 & 0 & 0 & 0 & 0 & 0.001 & 0 \\
\hline \multirow[t]{2}{*}{ Tbil $(\mu \mathrm{mol} / \mathrm{L})$} & 47.40 & 18.70 & 19.80 & 20.30 & 16.40 & 16.20 & 21.75 & 18.40 & 16.05 \\
\hline & $\begin{array}{l}(32.30- \\
69.80)\end{array}$ & $\begin{array}{l}(13.30- \\
26.90)\end{array}$ & $\begin{array}{l}(14.20- \\
28.40)\end{array}$ & $\begin{array}{l}(13.25- \\
26.70)\end{array}$ & $\begin{array}{l}(12.00- \\
21.40)\end{array}$ & $(11.30-28.00)$ & $(14.45--29.20)$ & $\begin{array}{l}(16.00- \\
23.00)\end{array}$ & $\begin{array}{l}(12.00- \\
22.40)\end{array}$ \\
\hline$P$ value & & 0 & 0 & 0 & 0 & 0 & 0 & 0 & 0 \\
\hline \multirow{2}{*}{$\begin{array}{l}\text { Albumin } \\
(\mathrm{g} / \mathrm{L})\end{array}$} & 32.80 & 32.60 & 33.00 & 31.70 & 31.50 & 33.20 & 31.90 & 33.00 & 33.50 \\
\hline & $\begin{array}{l}(30.70- \\
35.20)\end{array}$ & $\begin{array}{l}(29.70- \\
35.80)\end{array}$ & $\begin{array}{l}(30.00- \\
35.10)\end{array}$ & $\begin{array}{l}(29.50- \\
35.05)\end{array}$ & $\begin{array}{l}(29.70- \\
34.30)\end{array}$ & $(30.40-35.50)$ & $(30.30-36.80)$ & $\begin{array}{l}(31.60- \\
34.10)\end{array}$ & $\begin{array}{l}(31.90- \\
36.50)\end{array}$ \\
\hline$P$ value & & 0.684 & 0.875 & 0.266 & 0.274 & 0.752 & 0.774 & 0.949 & 0.092 \\
\hline \multirow{2}{*}{$\begin{array}{l}\text { Creatinine } \\
(\mathrm{mg} / \mathrm{dL})\end{array}$} & 75.50 & 64.00 & 63.00 & 53.00 & 51.50 & 62.50 & 61.00 & 66.50 & 61.00 \\
\hline & $\begin{array}{l}(61.00- \\
103.00)\end{array}$ & $\begin{array}{l}(53.00- \\
76.00)\end{array}$ & $\begin{array}{l}(56.00- \\
86.00)\end{array}$ & $\begin{array}{l}(45.00- \\
62.50)\end{array}$ & $\begin{array}{l}(47.00- \\
65.00)\end{array}$ & $(51.00-95.00)$ & $(51.50-77.00)$ & $\begin{array}{l}(55.00- \\
80.00)\end{array}$ & $\begin{array}{l}(49.00- \\
80.00)\end{array}$ \\
\hline$P$ value & & 0 & 0.018 & 0 & 0 & 0.064 & 0.007 & 0.084 & 0.001 \\
\hline \multirow{2}{*}{$\begin{array}{l}\text { WBC } \\
\left(\times 10^{9} / \mathrm{L}\right)\end{array}$} & 6.50 & 2.80 & 3.60 & 3.20 & 3.00 & 4.30 & 2.80 & 3.70 & 3.05 \\
\hline & $\begin{array}{l}(5.10- \\
8.20)\end{array}$ & $\begin{array}{l}(1.80- \\
4.80)\end{array}$ & $\begin{array}{l}(2.50- \\
5.40)\end{array}$ & $\begin{array}{l}(2.00- \\
4.50)\end{array}$ & $\begin{array}{l}(1.70- \\
5.60)\end{array}$ & $(2.30-6.00)$ & $(1.90-3.90)$ & $\begin{array}{l}(2.30- \\
4.60)\end{array}$ & $(2.00-4.40)$ \\
\hline$P$ value & & 0 & 0 & 0 & 0 & 0 & 0 & 0.001 & 0 \\
\hline \multirow{2}{*}{$\begin{array}{l}\text { Platelets } \\
\left(\times 10^{9} / \mathrm{L}\right)\end{array}$} & 95.00 & 57.00 & 65.00 & 72.50 & 74.00 & 111.00 & 44.00 & 70.50 & 62.00 \\
\hline & $\begin{array}{l}(71.00- \\
126.00)\end{array}$ & $\begin{array}{l}(37.00- \\
96.00)\end{array}$ & $\begin{array}{l}(47.00- \\
108.00)\end{array}$ & $\begin{array}{l}(55.00- \\
96.50)\end{array}$ & $\begin{array}{l}(55.00- \\
92.00)\end{array}$ & $(46.00-168.00)$ & $(35.00-70.00)$ & $\begin{array}{l}(37.00- \\
140.00)\end{array}$ & $\begin{array}{l}(40.00- \\
91.00)\end{array}$ \\
\hline$P$ value & & 0 & 0 & 0 & 0.006 & 0.584 & 0 & 0.257 & 0 \\
\hline Pre-TIPS OHE & $\begin{array}{l}8 \\
(5.48 \%)\end{array}$ & $\begin{array}{l}9 \\
(2.46 \%)\end{array}$ & $\begin{array}{l}4 \\
(5.80 \%)\end{array}$ & 2 & $\begin{array}{l}0 \\
(0.00 \%)\end{array}$ & $1(3.70 \%)$ & $1(3.45 \%)$ & $0(0.00 \%)$ & $0(0.00 \%)$ \\
\hline$P$ value & & 0.578 & 1 & 0.727 & 0.354 & 1 & 1 & 1 & 0.202 \\
\hline $\begin{array}{l}\text { Emergency } \\
\text { TIPS }\end{array}$ & $\begin{array}{l}1 \\
(0.68 \%)\end{array}$ & $\begin{array}{l}27 \\
(7.38 \%)\end{array}$ & $\begin{array}{l}6 \\
(8.70 \%)\end{array}$ & $\begin{array}{l}8 \\
(12.50 \%)\end{array}$ & $\begin{array}{l}5 \\
(16.67 \%)\end{array}$ & $2(7.41 \%)$ & $3(10.34 \%)$ & $1(7.14 \%)$ & $3(7.14 \%)$ \\
\hline$P$ value & & 0.572 & 0.005 & 0 & 0.001 & 0.064 & 0.015 & 0.168 & 0.035 \\
\hline $\begin{array}{l}\text { Pre-TIPS } \\
\text { splenectomy }\end{array}$ & $\begin{array}{l}0 \\
(0.00 \%)\end{array}$ & $\begin{array}{l}83 \\
(22.68 \%)\end{array}$ & $\begin{array}{l}10 \\
(14.49 \%)\end{array}$ & $\begin{array}{l}4 \\
(6.25 \%)\end{array}$ & $\begin{array}{l}2 \\
(6.67 \%)\end{array}$ & $11(40.74 \%)$ & $5(17.24 \%)$ & $\begin{array}{l}3 \\
(21.43 \%)\end{array}$ & 7 (16.67\%) \\
\hline$P$ value & & 0.347 & 0 & 0.008 & 0.028 & 0 & 0 & 0.001 & 0 \\
\hline Pre-TIPS PVT & $\begin{array}{l}19 \\
(13.01 \%)\end{array}$ & $\begin{array}{l}122 \\
(33.33 \%)\end{array}$ & $\begin{array}{l}23 \\
(33.33 \%)\end{array}$ & $\begin{array}{l}15 \\
(23.44 \%)\end{array}$ & $\begin{array}{l}5 \\
(16.67 \%)\end{array}$ & $14(51.85 \%)$ & 7 (24.14\%) & $\begin{array}{l}5 \\
(35.71 \%)\end{array}$ & $\begin{array}{l}12 \\
(28.57 \%)\end{array}$ \\
\hline $\begin{array}{l}\text { Abbreviations } \\
\text { disease; HBV, } \\
\text { hepatic encep } \\
\text { portal pressur } \\
\text { bilirubin; TIPS }\end{array}$ & ais & hep & $\mathrm{LT}$, al & minot & erase; & spartate amino & ferase; Crypt & $\begin{array}{l}\text { nic, crypto } \\
\text { iver diseas } \\
\text { ary biliary }\end{array}$ & $\begin{array}{l}\text { ic liver } \\
\text { HE, overt } \\
\text { hosis; PPG, } \\
\text { total }\end{array}$ \\
\hline
\end{tabular}




\begin{tabular}{|c|c|c|c|c|c|c|c|c|c|}
\hline & PA-HSOS & HBV & Alcohol & PBC & AlH & Schistosomiasis & $\mathrm{HCV}$ & Mixed & Cryptogenic \\
\hline$P$ value & & 0.324 & 0 & 0.059 & 0.567 & 0 & 0.151 & 0.039 & 0.031 \\
\hline \multirow[t]{2}{*}{$\begin{array}{l}\text { Pre-TIPS PVV } \\
(\mathrm{cm} / \mathrm{s})\end{array}$} & $\begin{array}{l}15.18 \pm \\
6.39\end{array}$ & $\begin{array}{l}25.61 \pm \\
8.99\end{array}$ & $\begin{array}{l}25.34 \pm \\
10.41\end{array}$ & $\begin{array}{l}24.57 \pm \\
6.82\end{array}$ & $\begin{array}{l}23.54 \pm \\
7.05\end{array}$ & $22.06 \pm 10.08$ & $25.92 \pm 8.47$ & $\begin{array}{l}19.25 \pm \\
11.13\end{array}$ & $\begin{array}{l}23.64 \pm \\
10.66\end{array}$ \\
\hline & & 0 & 0 & 0 & 0 & 0.009 & 0 & 0.339 & 0 \\
\hline \multicolumn{10}{|l|}{ Stent diameter } \\
\hline $6 \mathrm{~mm}$ & $\begin{array}{l}4 \\
(2.74 \%)\end{array}$ & $\begin{array}{l}62 \\
(16.94 \%)\end{array}$ & $\begin{array}{l}11 \\
(15.94 \%)\end{array}$ & $\begin{array}{l}19 \\
(29.69 \%)\end{array}$ & $\begin{array}{l}9 \\
(30.00 \%)\end{array}$ & $3(11.11 \%)$ & $11(37.93 \%)$ & $0(0.00 \%)$ & $\begin{array}{l}12 \\
(28.57 \%)\end{array}$ \\
\hline $7 \mathrm{~mm}$ & $\begin{array}{l}16 \\
(10.96 \%)\end{array}$ & $\begin{array}{l}36 \\
(9.84 \%)\end{array}$ & $\begin{array}{l}6 \\
(8.70 \%)\end{array}$ & $\begin{array}{l}3 \\
(4.69 \%)\end{array}$ & $\begin{array}{l}3 \\
(10.00 \%)\end{array}$ & $3(11.11 \%)$ & $1(3.45 \%)$ & $0(0.00 \%)$ & $4(9.52 \%)$ \\
\hline $8 \mathrm{~mm}$ & $\begin{array}{l}126 \\
(86.30 \%)\end{array}$ & $\begin{array}{l}268 \\
(73.22 \%)\end{array}$ & $\begin{array}{l}52 \\
(75.36 \%)\end{array}$ & $\begin{array}{l}42 \\
(65.63 \%)\end{array}$ & $\begin{array}{l}18 \\
(60.00 \%)\end{array}$ & 21 (77.78\%) & $17(58.62 \%)$ & $\begin{array}{l}14 \\
(100.00 \%)\end{array}$ & $\begin{array}{l}26 \\
(61.90 \%)\end{array}$ \\
\hline$P$ value & & 0.044 & 0.002 & 0 & 0 & 0.126 & 0 & 0.334 & 0 \\
\hline $\begin{array}{l}\text { Preoperative } \\
\text { PVP }(\mathrm{mmHg})\end{array}$ & $\begin{array}{l}29.19 \pm \\
4.75\end{array}$ & $\begin{array}{l}29.22 \pm \\
5.87\end{array}$ & $\begin{array}{l}31.10 \pm \\
5.75\end{array}$ & $\begin{array}{l}29.82 \pm \\
5.77\end{array}$ & $\begin{array}{l}29.03 \pm \\
3.99\end{array}$ & $29.08 \pm 4.68$ & $29.89 \pm 4.60$ & $\begin{array}{l}29.19 \pm \\
6.97\end{array}$ & $\begin{array}{l}30.41 \pm \\
7.55\end{array}$ \\
\hline$P$ value & & 0.962 & 0.015 & 0.431 & 0.865 & 0.912 & 0.496 & 1 & 0.243 \\
\hline $\begin{array}{l}\text { Postoperative } \\
\text { PVP (mmHg) }\end{array}$ & $\begin{array}{l}17.63 \pm \\
4.72\end{array}$ & $\begin{array}{l}20.36 \pm \\
5.27\end{array}$ & $\begin{array}{l}21.79 \pm \\
5.97\end{array}$ & $\begin{array}{l}20.61 \pm \\
6.09\end{array}$ & $\begin{array}{l}20.14 \pm \\
4.11\end{array}$ & $18.17 \pm 5.71$ & $20.29 \pm 5.92$ & $\begin{array}{l}22.53 \pm \\
6.23\end{array}$ & $\begin{array}{l}19.73 \pm \\
5.57\end{array}$ \\
\hline$P$ value & & 0 & 0 & 0.001 & 0.011 & 0.606 & 0.039 & 0.001 & 0.018 \\
\hline $\begin{array}{l}\text { PPG } \\
\text { reduction } \\
\text { (mmHg) }\end{array}$ & $\begin{array}{l}14.54 \pm \\
5.97\end{array}$ & $\begin{array}{l}9.59 \pm \\
5.48\end{array}$ & $\begin{array}{l}10.02 \pm \\
5.68\end{array}$ & $\begin{array}{l}10.26 \pm \\
5.92\end{array}$ & $\begin{array}{l}10.01 \pm \\
4.98\end{array}$ & $9.91 \pm 6.19$ & $10.05 \pm 6.37$ & $\begin{array}{l}9.16 \pm \\
7.74\end{array}$ & $\begin{array}{l}13.92 \pm \\
6.58\end{array}$ \\
\hline$P$ value & & 0 & 0 & 0 & 0 & 0.002 & 0.001 & 0.004 & 0.586 \\
\hline
\end{tabular}

\section{Comparison of orthotopic liver transplantation-free survival between PA-HSOS patients and patients with other causes of portal hypertension}

The median follow-up time of PA-HSOS and patients with other causes of portal hypertension was 16.48 (10.17-26.76) and 22.10 (11.23-41.08) months, respectively. During the follow-up period, 165 patients died or underwent liver transplantation. The primary causes of death are listed in Table 2. Half of the deaths in the PA-HSOS patients were caused by liver failure, and no PA-HSOS patients died as a result of malignancy or bleeding. Four patients had biliary tract hemorrhage associated with the TIPS procedure. Although three of them were treated initially successfully with endoscopic retrograde cholangiopancreatography, they eventually died of liver failure. 
Table 2

Primary causes of death in patients with portal hypertension $(n=165)$ of different etiology after TIPS

\begin{tabular}{|c|c|c|c|c|c|c|c|c|c|}
\hline & PA-HSOS & HBV & Alcohol & PBC & AlH & Schistosomiasis & $\mathrm{HCV}$ & Mixed & Cryptogenic \\
\hline & $(n=22)$ & $(n=78)$ & $(n=14)$ & $(n=15)$ & $(n=6)$ & $(n=9)$ & $(n=11)$ & $(n=4)$ & $(n=6)$ \\
\hline Liver failure & 11 & 19 & 3 & 7 & 2 & 2 & 0 & 0 & 1 \\
\hline Sepsis or infection & 3 & 2 & 1 & 0 & 0 & 1 & 2 & 0 & 2 \\
\hline Hemorrhage & 0 & 15 & 2 & 1 & 1 & 3 & 3 & 2 & 0 \\
\hline $\mathrm{HE}$ & 1 & 6 & 1 & 1 & 1 & 1 & 0 & 1 & 1 \\
\hline $\mathrm{HCC}$ & 0 & 12 & 0 & 0 & 0 & 0 & 3 & 1 & 0 \\
\hline MODS & 6 & 8 & 2 & 1 & 0 & 0 & 0 & 0 & 2 \\
\hline Not liver related & 1 & 8 & 1 & 2 & 1 & 2 & 1 & 0 & 0 \\
\hline Unknown & 0 & 2 & 1 & 1 & 0 & 0 & 1 & 0 & 0 \\
\hline Transplant & 0 & 6 & 3 & 2 & 1 & 0 & 1 & 0 & 0 \\
\hline
\end{tabular}

Abbreviations: AlH, autoimmune hepatitis; Cryptogenic, cryptogenic liver disease; HBV, hepatitis B virus; HCV, hepatitis C virus; HCC, hepatocellular carcinoma; HE, hepatic encephalopathy; MODS, Multiple Organ Dysfunction Syndrome; PA-HSOS, pyrrolizidine alkaloid-induced hepatic sinusoidal obstruction syndrome; PBC, primary biliary cirrhosis

The Kaplan-Meier survival curves are shown in Fig. 2. No significant differences were found in the orthotopic liver transplantation (OLT)-free survival between patients with PA-HSOS and patients with other causes of portal hypertension (all $P>0.05$ ).

In the univariate and multivariate analyses, factors influencing the OLT-free survival of PA-HSOS patients included the MELD score, severity of ascites, ALT, AST, Tbil, and creatinine serum levels, and the pre-TIPS PVV. In the multivariate analysis, the MELD score, AST, Tbil, and creatinine serum levels, and pre-TIPS PPV remained significant risk factors (Online Resource 1). In patients with other portal hypertension etiologies, age, the MELD and CTP scores, severity of ascites, PT, INR, Tbil, albumin concentration, PLT, pre-TIPS overt hepatic encephalopathy (OHE), pre-TIPS portal vein thrombosis, pre-TIPS PVV, pre-TIPS PVP (portal vein pressure), and post-operative PVP were significantly associated with OLT-free survival in the univariate analysis. The Cox proportional hazard model showed that age, CTP score, INR, Tbil, albumin, and pre-TIPS PVV were independent prognostic markers for OLT-free survival in patients with various etiologies of portal hypertension (Online Resource 2).

\section{Comparison of OLT-free survival between PA-HSOS patients and patients with other causes of portal hypertension after propensity score matching}

In view of the significant differences in the baseline characteristics between the patients with different etiologies of portal hypertension, we performed a 1:1 propensity score matching (PSM) analysis. Using PSM, PA-HSOS patients and patients with other portal hypertension etiologies were matched for age, sex, MELD score, CTP score, stent diameter, and pre-TIPS episodes of OHE. The above-mentioning matching variables were more balanced after PSM as compared to before (Online Resources 3-7). In general, PA-HSOS patients had a statistically significantly better or similar prognosis than/as patients with other etiologies of portal hypertension. PA-HSOS patients had a significantly longer OLT-free survival time than HBV/HCV patients [PA-HSOS: 17.68 (10.28-27.12) months vs HBV/HCV: 16.18 (8.02-33.77) months; $P=0.0011]$ (Fig. 3a). PA-HSOS patients had a statistically significantly longer OLT-free survival time [19.20 (10.87-24.33) months] as compared to those of primary biliary cirrhosis and autoimmune liver hepatitis patients [20.55 (11.28-36.23) months; $P=0.021]$ (Fig. 3c). Patients with PA-HSOS had a significantly longer OLT-free survival time [16.72 (8.90-27.63) months] than patients with schistosomiasis infection [14.72 (8.17-20.07) months; $P=0.025]$ (Fig. 3d). However, OLT-free survival was compared between PA-HSOS patients and alcoholic liver disease patients and did not differ significantly by Kaplan-Meier [PAHSOS: 13.78 (10.17-25.53) months vs alcoholic liver disease: 21.17 (9.67-37.40) months; $P=0.31]$ (Fig. 3b). Similarly, when compared to mixed/cryptogenic etiology of portal hypertension patients, OLT-free survival did not differ by Kaplan-Meier [PA-HSOS: 13.13 (9.70-23.53) months vs mixed/cryptogenic etiology of portal hypertension : 17.27 (9.80-28.47) months; $P=0.48]$ (Fig. 3e).

\section{Discussion}

Our data indicate that TIPS results in better or similar survival rates in patients with PA-HSOS as compared to those of patients with other etiologies of portal hypertension.

As compensated cirrhosis decompensates, a number of complications can occur, among which complications related to portal hypertension are a common cause of death $[22,23]$. 
Although pharmacological and endoscopic therapies have been widely introduced, the TIPS procedure is considered the most effective and fastest option for lowering portal venous pressure [22]. We hypothsized that the different etiologies of portal hypertension with their underlying pathogenic mechanisms may result in different long-term survival after TIPS. While a few retrospective studies found no significant differences in the prognosis of patients with portal hypertension of different etiologies, none of these studies included HSOS patients [24-26].

In our study, the OLT-free survival rate for PA-HSOS patients was $86.5 \%$ during the follow-up period, which was comparable to the findings of other studies (64.3-94.6\%) [10,11, 27]. After accounting for confounding factors using PSM, TIPS achieved a significantly better OLT-free survival as compared to that in patients with viral hepatitis, with primary biliary cirrhosis and autoimmune liver hepatitis patients, and with schistosomiasis infection. Careful patient selection remains a critical factor that determines patient outcomes after TIPS, and high CTP and MELD scores have been associated with OHE and high mortality after TIPS treatment [21, 28].

In this study, PA-HSOS patients had higher CTP and MELD scores than patients with other etiologies of portal hypertension at baseline. However, remarkably, these patients had a better or similar survival than patients with different other etiologies of portal hypertension after TIPS treatment. This may have different explanations. First, PA-HSOS patients seldom present with gastroesophageal varices and splenomegaly because of the rapid progression of their portal hypertension. Therefore, these patients have a lower risk of dying from massive gastrointestinal bleeding both before and after TIPS treatment [11]. Second, early during PA-HSOS, liver damage is mostly mild to moderate, whereas the abrupt occurrence of severe portal hypertension without effective compensation is seen as the main cause of the occurrence of clinical symptoms and indicate a poor prognosis [11]. TIPS may benefit patients with PA-HSOS through the prompt decompression of the portal vein [22]. Finally, as an acute druginduced liver injury, liver damage in PA-HSOS patients usually does not progress once PA intake is stopped. This is reflected in the rapid decrease in blood pyrrole-protein adducts as biomarkers of PA-induced liver injury, and indicates that the liver damage may be repaired, which favors the longterm survival of patients treated with TIPS [29].

In our study, the MELD score, AST, Tbil, and creatinine serum levels, and pre-TIPS PVV were predictors of survival in PA-HSOS patients. Several studies have also demonstrated that serum bilirubin concentration is independently related to survival in PA-HSOS patients [2, 10]. Furthermore, we identified the following six factors that were independently associated with survival in patients with other etiologies of portal hypertension: age, CTP score, INR, Tbil and albumin serum levels, and pre-TIPS PVV. Age above 70 years has been reported to be associated with reduced survival within 90 days following TIPS [30]. INR and Tbil and albumin levels are also important factors included in the CTP and MELD scores.

In total, 22 PA-HSOS patients died during the follow-up period. Of note, 11 patients died one month after TIPS because of sepsis ( $\mathrm{n}=1$ ), heart failure $(n=1)$, multiple organ dysfunction syndrome $(n=5)$, or liver failure $(n=4)$. There were significant differences in the MELD score at baseline between patients who died within one month after TIPS and patients who died at least 1 month after TIPS $(22.18 \pm 7.51$ vs. $15.36 \pm 6.53, P=$ 0.034). Therefore, we recommend paying particular attention to the monitoring of PA-HSOS patients during the first month postoperatively if we are to improve survival rates in these patients.

Our results should be interpreted within the limitations of the study. First, this was a retrospective single-center study. However, this facilitates quality control of the TIPS procedure because all procedures were performed by the same experienced group. Second, the heterogeneity of baseline characteristics among the patients with different etiologies of portal hypertension might have resulted in bias. Although we accounted for confounding factors with PSM, several factors remained incomparable. Third, we did not evaluate the shunt dysfunction rate and post-intervention encephalopathy rate for these parameters had not been recorded in the patients' medical files. Finally, PSM significantly reduced the sample size of the analyzed cohort. Therefore, we combined HBV with HCV patients, primary biliary cirrhosis patients with autoimmune hepatitis patients, and patients with mixed etiologies of portal hypertension with cryptogenic portal hypertension patients in three new subgroups.

In summary, TIPS placement can be performed safely in patients with PA-HSOS to treat portal hypertension. The OLT-free survival of PA-HSOS patients after TIPS is better or similar to that of patients with various other causes of portal hypertension. Large, randomized, multicenter, prospective studies are needed to verify our findings.

\section{Declarations}

\section{Ethics approval and consent to participate}

This study was approved by the ethics committee of the Nanjing Drum Tower Hospital.

The ethics committee of the Nanjing Drum Tower Hospital waived the need for consent because of the retrospective and anonymized nature of the study. All methods were carried out in accordance with relevant guidelines and regulations. All procedures followed were in accordance with the ethical standards of the responsible committee on human experimentation (institutional and national) and with the Helsinki Declaration of 1964, as revised in 2013.

\section{Consent for publication}


Not applicable.

\section{Availability of data and materials}

The datasets used and/or analysed during the current study are available from the corresponding author on reasonable request.

\section{Competing interests}

The authors declare that they have no competing interests.

\section{Funding}

This research received no specific grant from any funding agency in the public, commercial, or not-for-profit sectors.

\section{Authors' contributions}

Chen YR analyzed and interpreted the patient data, and was a major contributor in writing the manuscript. Zhang W designed the work. Zhang M performed the transjugular intrahepatic portosystemic shunt placement and collected the patient data. Zhang $\mathrm{F}$ performed the transjugular intrahepatic portosystemic shunt placement and collected the patient data. Xiao JQ performed the transjugular intrahepatic portosystemic shunt placement and collected the patient data. Yin Q collected and analyzed the patient data. Zhuge YZ designed the study and interpreted the data. All authors read and approved the final manuscript.

\section{Acknowledgements}

Not applicable.

\section{References}

1. Zhuge Y, Liu Y, Xie W, et al. Expert consensus on the clinical management of pyrrolizidine alkaloid-induced hepatic sinusoidal obstruction syndrome. J Gastroenterol Hepatol. 2019; 34:634-642.

2. Wang X, Qi X, Guo X. Tusanqi-Related Sinusoidal Obstruction Syndrome in China: A Systematic Review of the Literatures. Medicine (Baltimore). 2015; 94(23): e942-e952.

3. Strouse C, Zhang Y, Zhang MJ, DiGilio A, et al. Risk Score for the Development of Veno-Occlusive Disease after Allogeneic Hematopoietic Cell Transplant. Biol Blood Marrow Transplant. 2018; 24(10):2072-2080.

4. DeLeve LD, Shulman HM, McDonald GB. Toxic injury to hepatic sinusoids: sinusoidal obstruction syndrome (venoocclusive disease). Semin Liver Dis. 2002; 22: 27-42

5. Mohty M, Malard F, Abecassis M, et al. Revised diagnosis and severity criteria for sinusoidal obstruction syndrome/veno-occlusive disease in adult patients: a new classification from the European Societyfor Blood and Marrow Transplantation. Bone Marrow Transplant. 2016; 51:90612.

6. Liu F, Rong X, Guo H, et al. Clinical characteristics,CT signs,and pathological findings of Pyrrolizidine alkaloids-induced sinusoidal obstructive syndrome: a retrospective study. BMC Gastroenterol. 2020; 20:30-39.

7. Ravaioli F,Colecchia A,Alemanni LV.Role of imaging techniques in liver veno-occlusive disease diagnosis: recent advances and literature review. Expert Rev Gastroenterol Hepatol. 2019; 13:463-484.

8. Siramolpiwat S. Transjugular intrahepatic portosystemic shunts and portal hypertension-related complications. World J Gastroenterol. 2014; 20:16996-7010.

9. Peng CY, Zhang XF, Zhang F, et al. Clinical efficacy and safety of anticoagulation therapy for Pyrrolizidine alkaloids-induced hepatic sinusoidal obstruction syndrome: a retrospective multicenter cohort study. Eur J Gastroenterol Hepatol. 2020; 32: 1168-1178.

10. Wang Y, Qiao D, Li Y, Xu F. Risk factors for hepatic veno-occlusive disease caused by Gynura segetum:A retrospective study. BMC Gastroenterol. 2018; 18: 156-163.

11. Zhuge YZ, Wang Y, Zhang F, et al. Clinical characteristics and treatment of pyrrolizidine alkaloid-related hepatic vein occlusive disease. Liver Int. 2018; 38: 1867-1874

12. European Association for the Study of the Liver. EASL 2017 Clinical Practice Guidelines on the management of hepatitis B virus infection. $J$ Hepatol. 2017;67:370-398. 
13. European Association for the Study of the Liver. EASL Clinical Practice Guidelines: Management of alcohol-related liver disease. J Hepatol. 2018;69: 154-181.

14. European Association for the Study of the Liver. EASL Clinical Practice Guidelines: The diagnosis and management of patients with primary biliary cholangitis. J Hepatol. 2017; 67: 145-172.

15. European Association for the Study of the Liver. EASL Clinical Practice Guidelines: Autoimmune hepatitis. J Hepatol. 2015; 63: $971-1004$.

16. Wu W, Feng A, Huang Y. Research and control of advanced schistosomiasis japonica in China. Parasitol Res. 2015;114:17-27.

17. European Association for Study of Liver. EASL Clinical Practice Guidelines: management of hepatitis C virus infection. J Hepatol. 2014; 60:392-420.

18. Mercado-Irizarry A, Torres EA. Cryptogenic cirrhosis: Current knowledge and future directions. Clin Liver Dis (Hoboken). $2016 ; 7: 69-72$.

19. De Franchis R, Baveno VIF. Expanding consensus in portal hypertension: report of the Baveno VI Consensus Workshop: stratifying risk and individualizing care for portal hypertension. J Hepatol. 2015; 63: 743-

20. Leng XF, Zhang F, Zhang M, et al. Comparison of transjugular intrahepatic portosystemic shunt for treatment of variceal bleeding in patients with cirrhosis with or without spontaneous portosystemic shunt. Eur J Gastroenterol Hepatol. 2019; 31:853-858.

21. Yin $X$, Zhang F, Guo H, et al. A nomogram to predict the risk of hepatic encephalopathy after transjugular intrahepatic portosystemic shunt in Cirrhotic Patients. Sci Rep. 2020; 10:9381-9383.

22. Strunk H, Marinova M. Transjugular Intrahepatic Portosystemic Shunt (TIPS): Pathophysiologic Basics, Actual Indications and Results with Review of the Literature. Rofo. 2018; 190:701-711.

23. Parker R. Role of transjugular intrahepatic portosystemic shunt in the management of portal hypertension. Clin Liver Dis. 2014; 18:319-34.

24. Perello MP, Mur JP, Vives MS, et al. Long-term follow-up of transjugular intrahepatic portosystemic shunt (TIPS) with stent-graft. Diagn Interv Radiol. 2019; 25:346-352.

25. Tripathi D, Ferguson J, Barkell H, et al. Improved clinical outcome with transjugular intrahepatic portosystemic stent-shunt utilizing polytetrafluoroethylene-covered stent. Eur J Gastroenterol Hepatol. 2006;18:225-32.

26. Vignali C, Bargellini I, Grosso M, et al. TIPS with expanded polytetrafluoroethylene-covered stent: results of an Italian multicenter study. AJR Am J Roentgenol. 2005; 185:472-80.

27. Zhou CZ, Wang RF, Lv WF, et al. Transjugular intrahepatic portosystemic shunt for pyrrolizidine alkaloid-related hepatic sinusoidal obstruction. World J Gastroenterol. 2020; 26:3472-3483.

28. Flud CR, Duarte-Rojo A. Prognostic Implications of Minimal/Covert Hepatic Encephalopathy: Large-scale Validation Cohort Studies. J Clin Exp Hepatol 2019; 9:112-116.

29. Gao H, Ruan JQ, Chen J, et al. Blood pyrrole-protein adducts as a diagnostic and prognostic index in pyrrolizidine alkaloid-hepatic sinusoidal obstruction syndrome. Drug Des Devel Ther. 2015;25:4861-4868.

30. Saad N, Rude MK, Darcy M, Hanin JB, Wentworth A, Korenblat KM. Older age is associated with increased early mortality after transjugular intrahepatic portosystemic shunt. Ann Hepatol. 2016; 15:215-221.

\section{Figures}




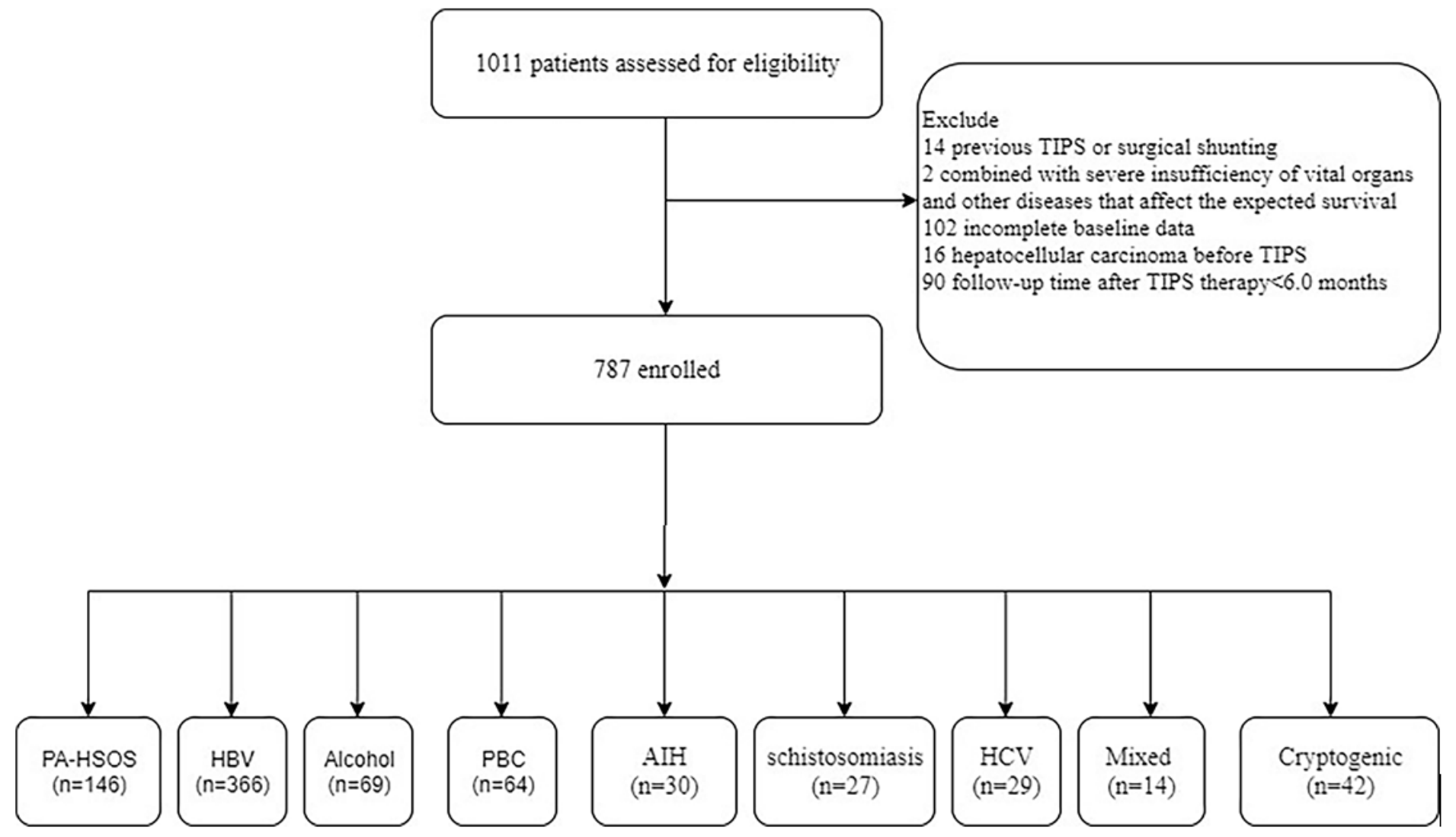

\section{Figure 1}

Flow chart of patients with portal hypertension of different etiologies ( $n=1011)$ through this study AlH, autoimmune hepatitis; HBV, hepatitis-B virus; HCV, hepatitis-C virus; PA-HSOS, pyrrolizidine alkaloids-induced hepatic sinusoidal obstruction syndrome; PBC, primary biliary cirrhosis

$$
\text { Strata + } \mathrm{HBV}+\mathrm{PBC}+\text { Schistosomiasis + Mixed + PA-HSOS }
$$

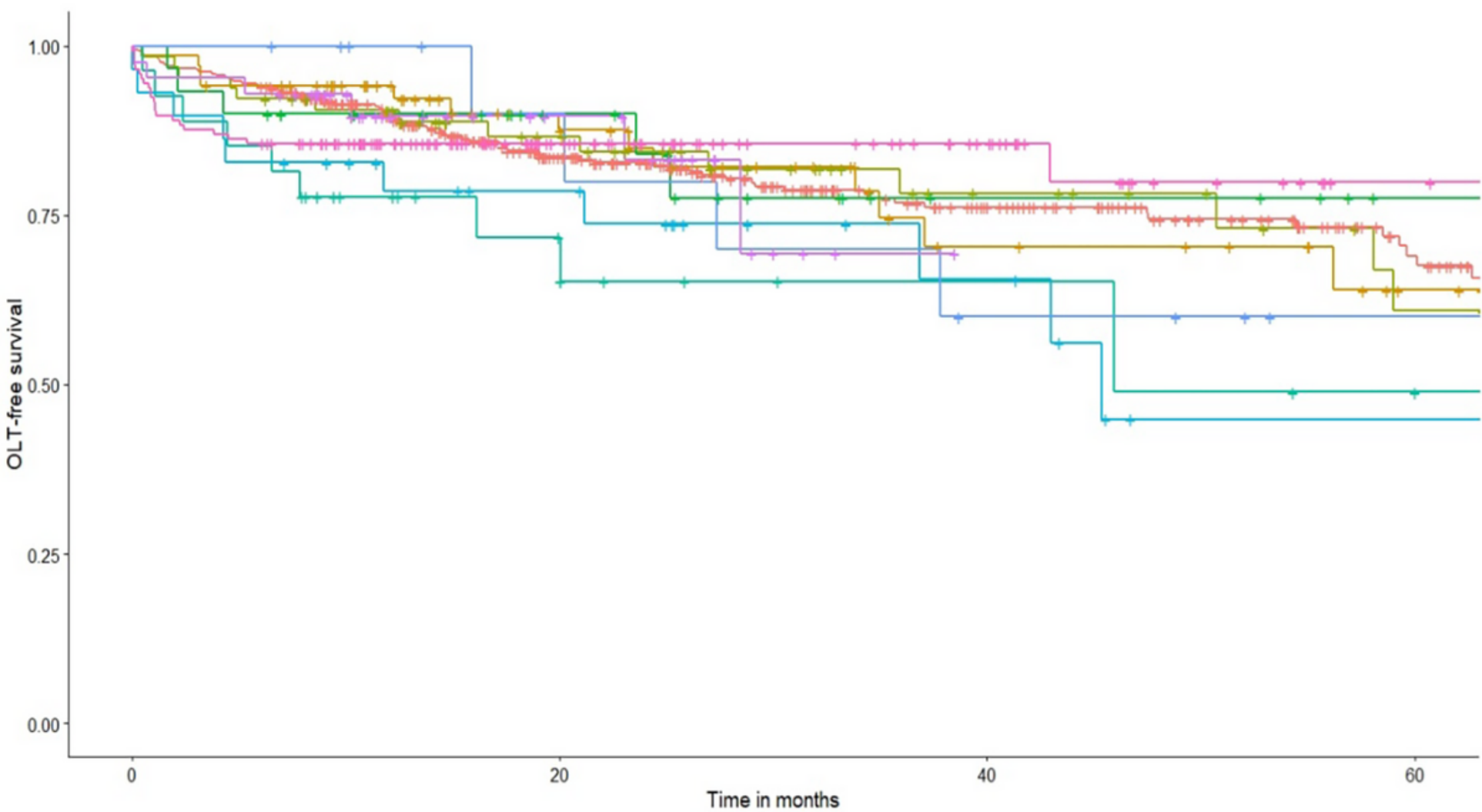


Figure 2

Comparison of orthotopic liver transplantation-free survival between portal hypertension patients with different etiologies $\mathrm{AlH}$, autoimmune liver hepatitis; Cryptogenic, cryptogenic liver disease; HBV, hepatitis-B virus; HCV, hepatitis-C virus; PA-HSOS, pyrrolizidine alkaloids-induced hepatic sinusoidal obstruction syndrome; PBC, primary biliary cirrhosis.
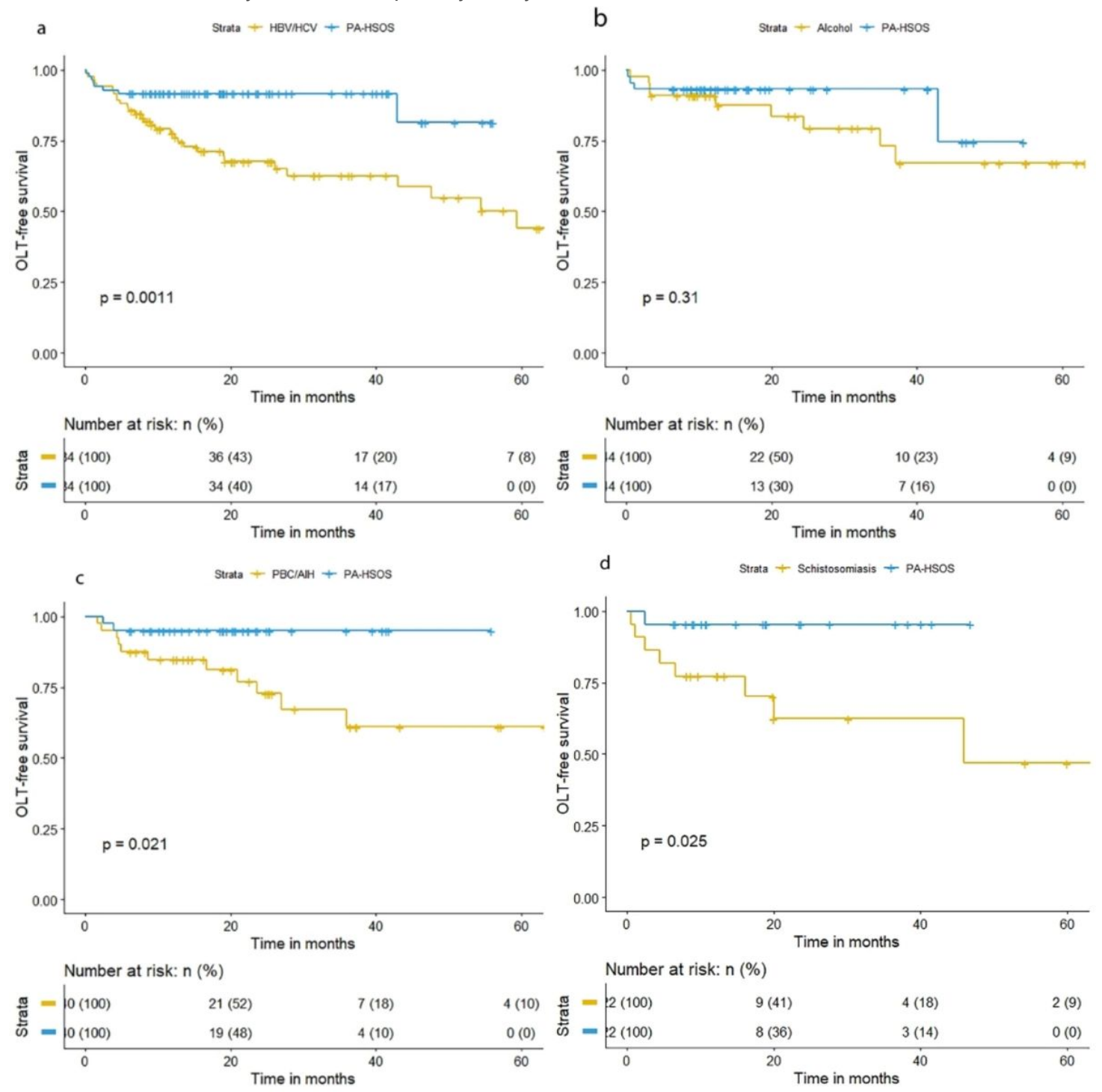

\section{Figure 3}

Kaplan-Meier curves after propensity score matching compared between PA-HSOS patients and a) HBV/HCV patients; b) alcoholic liver disease patients; c) primary biliary cirrhosis and autoimmune hepatitis patients d) schistosomiasis patients e) mixed/cryptogenic etiology of portal hypertension patients $\mathrm{AlH}$, autoimmune hepatitis; HBV, hepatitis-B virus; HCV, hepatitis-C virus; PA-HSOS, pyrrolizidine alkaloids-induced hepatic sinusoidal obstruction syndrome; $\mathrm{PBC}$, primary biliary cirrhosis

\section{Supplementary Files}

This is a list of supplementary files associated with this preprint. Click to download.

- SupplementFile1.docx 